Research Article

\title{
On Periodic Dividends for the Classical Risk Model with Debit Interest
}

\author{
Hua Dong $(\mathbb{D}$ and Xianghua Zhao \\ School of Statistics, Qufu Normal University, Shandong 273165, China \\ Correspondence should be addressed to Hua Dong; sddh1978@126.com
}

Received 13 January 2020; Accepted 11 February 2020; Published 17 March 2020

Guest Editor: Zhimin Zhang

Copyright (c) 2020 Hua Dong and Xianghua Zhao. This is an open access article distributed under the Creative Commons Attribution License, which permits unrestricted use, distribution, and reproduction in any medium, provided the original work is properly cited.

A periodic dividend problem is studied in this paper. We assume that dividend payments are made at a sequence of Poisson arrival times, and ruin is continuously monitored. First of all, three integro-differential equations for the expected discounted dividends are obtained. Then, we investigate the explicit expressions for the expected discounted dividends, and the optimal dividend barrier is given for exponential claims. A similar study on a generalized Gerber-Shiu function involving the absolute time is also performed. To demonstrate the existing results, we give some numerical examples.

\section{Introduction}

Suppose the dynamics of the surplus process of an insurance company at time $t$ is defined as the solution to

$$
\mathrm{d} R_{t}= \begin{cases}c \mathrm{~d} t-\mathrm{d} S_{t}, & R_{t} \geq 0, \\ \left(c+\alpha R_{t}\right) \mathrm{d} t-\mathrm{d} S_{t}, & -\frac{c}{\alpha} \leq R_{t}<0,\end{cases}
$$

where $c>0$ is the premium charged in the unit time and $\alpha>0$ is the debit interest. $S_{t}=\sum_{i=1}^{N(t)} X_{i}$ is a compound Poisson process with intensity $\gamma>0$ representing the total claim amounts until time $t$, and $X_{i}$ is the $i$ - th claim size. Suppose that the claim sizes are independent of each other and have common density function $\varsigma$. We also assume that $X_{i}$ and $N(t)$ are mutually independent.

Model (1) means that the insurer can make loans at the rate $\alpha>0$ once the process $R_{t}$ goes below zero. When $R(t)<-c / \alpha$, the insurer can be prohibited to run its business, i.e., the insurer is absolutely ruined. The absolute ruin risk models have been investigated extensively, see Gerber and Yang [1], Cai [2], Yuen et al. [3], Wang and Yin [4], and Cai and Yang [5], among others.

In recent years, the research problems in risk theory are more and more closely related to real life. The risk model with debit interest is a good example. The insurer cannot monitor the surplus continuously, and the dividend can be paid at some certain times. Because of its importance in real life, the topic of periodic dividends has become very popular in risk theory during the last twenty years. Considering dividends can only be made at some discrete times in practice, Albrecher et al. [6] put forward the periodic barrier dividends in this type of risk model. They assumed that both barrier dividends and ruin can only be observed at some randomized times. Considering the insurer monitors bankruptcy more closely than dividends, Avanzi et al. [7] investigated periodic dividend barrier strategy in the dual model where the solvency is monitored continuously. For those risk models with periodic dividends and bankruptcy, the reader can refer to Zhang and Cheung [8,9], Avanzi et al. [10], Dong et al. [11], and Peng et al. [12], among others. Different to the papers mentioned above, the periodic dividend strategy in Peng et al. [12] is threshold strategy.

Now, we study surplus process (1) under periodic dividend strategy. We assume the ruin is continuously monitored as usual. Let $\left\{Z_{i}\right\}$ be the sequence of dividend observation times with $Z_{0}=0$ and $W_{i}=Z_{i}-Z_{i-1}(i=1,2, \ldots)$ be an exponentially random variable with $\mathbb{E} W_{i}=1 / \beta$. In addition, we assume that 
$W_{k}(k=1,2, \ldots)$ are independent of $N(t)$ and $X_{i}$. Furthermore, we assume that no dividends are made at time 0 . The modified surplus process is given by $R_{t}^{b}$ with $b>0$ being the constant level of the dividend barrier. Denote by $T=$ $\inf \left\{t>0: R_{t}^{b}<-(c / \alpha)\right\}$ the time of absolute ruin for $R_{t}^{b}$ with the convention that inf $\phi=\infty$.

Let

$$
v(x, b)=\mathbb{E}_{x}\left[\sum_{Z_{k} \leq T} e^{-\sigma Z_{k}}\left(R_{Z_{k}}^{b}-b\right)_{+}\right],
$$

be the expected discounted dividend before time $T$, and $\sigma \geq 0$. The generalized Gerber-Shiu function is also our concern:

$$
\Phi(x)=\mathbb{E}_{x}\left[e^{-\sigma T} s^{N(T)} \omega\left(R_{T-}^{b},\left|R_{T}^{b}\right|\right) 1_{\{T<\infty\}}\right], \quad 0<s \leq 1,
$$

where $1_{A}$ denotes the indicator function of event $A$. $\omega(x, y) \geq 0$ is a bounded measurable function of $x \geq-(c / \alpha), y>(c / \alpha), R_{T-}^{b}$ is the surplus prior to time $T$, and $\left|R_{T}^{b}\right|$ is the deficit at time $T$. When $s=1, \Phi(x)$ is the Gerber-Shiu function proposed by Gerber and Shiu [13]. When $\omega(\cdot, \cdot)=1, \Phi(x)$ describes the joint distribution of the absolute time $T$ and $N(T)$ :

$$
\phi(x)=\mathbb{E}_{x}\left[s^{N(T)} e^{-\sigma T} 1_{\{T<\infty\}}\right] .
$$

When $\omega(\cdot, \cdot)=1, \sigma=0, \Phi(x)$ is the probability generating function of $N(T)$. The topic on the number of claims has been studied by many scholars in various forms. Some scholars focus on some generalized Gerber-Shiu functions, see Li and Lu [14] and Wang et al. [15]; some only considered the Laplace transform, see Egidio dos Reis [16]; and some studied the density function, see Dickson [17] and Czarna et al. [18].

We arrange the paper as follows. In Section 2, we first derive a system of integro-differential equations for $\nu(x, b)$. For exponential claims, explicit results for $\nu(x, b)$ are given. The generalized Gerber-Shiu function is discussed in Section 3. In Section 4, some numerical results are shown to illustrate the given results.

\section{Results for $v(x, b)$}

2.1. Integro-differential Equations for $v(x, b)$. The integrodifferential equation is a conventional method; we start this section with the expression for $v(x, b)$.

Theorem 1. For $-(c / \alpha) \leq x<0$, we have

$$
(\alpha x+c) v^{\prime}(x, b)-(\sigma+\gamma) v(x, b)+\gamma \int_{0}^{x+c / \alpha} v(x-z, b) \varsigma(z) \mathrm{d} z=0,
$$

for $0 \leq x<b$, we have

$$
c \nu^{\prime}(x, b)-(\sigma+\gamma) \nu(x, b)+\gamma \int_{0}^{x+c / \alpha} \nu(x-z, b) \varsigma(z) \mathrm{d} z=0
$$

and for $x>b$, we have

$$
\begin{aligned}
& c \nu^{\prime}(x, b)-(\sigma+\beta+\gamma) \nu(x, b)+\beta(x-b)+\beta \nu(b, b) \\
& \quad+\gamma \int_{0}^{x+c / \alpha} v(x-z, b) \varsigma(z) \mathrm{d} z=0 .
\end{aligned}
$$

In addition, we have the following relations:

$$
\begin{aligned}
& v(b-, b)=v(b+, b), \\
& v^{\prime}(b-, b)=v^{\prime}(b+, b), \\
& v(0-, b)=v(0+, b), \\
& v^{\prime}(0-, b)=v^{\prime}(0+, b), \\
& v\left(-\frac{c}{\alpha}, b\right)=0 .
\end{aligned}
$$

Proof. A standard method is used here, see also Yuen et al. [3] and Albrecher et al. [6]. For a small interval $(0, s)$, all the possible events are taken into account. Then, we have

$$
\begin{aligned}
\nu(x, b)= & e^{-(\beta+\sigma+\gamma) s} \nu(x+c s, b)+\beta s e^{-(\sigma+\gamma) s} \\
& \cdot[x+c s-b+\nu(b, b)] 1_{\{x+c s>b\}} \\
& +\beta s e^{-(\sigma+\gamma) s} \nu(x+c s, b) 1_{\{0<x+c s<b\}} \\
& +\gamma s e^{-(\sigma+\gamma) s} \int_{0}^{x+c s+c / \alpha} \nu(x+c s-y, b) \varsigma(y) \mathrm{d} y+o(s),
\end{aligned}
$$

for $x \geq 0$, and for $-(c / \alpha) \leq x<0$,

$$
\begin{aligned}
\nu(x, b)= & e^{-(\beta+\sigma+\gamma) s} \nu\left(t_{\alpha}(s, x), b\right)+\beta s e^{-(\sigma+\gamma) s} \nu\left(t_{\alpha}(s, x)\right) \\
& +\gamma s e^{-(\sigma+\beta) s} \int_{0}^{t_{\alpha}(s, x)+c / \alpha} \nu\left(t_{\alpha}(s, x)-y, b\right) \varsigma(y) \mathrm{d} y+o(s),
\end{aligned}
$$

where $t_{\alpha}(s, x)=x e^{\alpha s}+c\left(e^{\alpha s}-1\right) / \alpha$.

Taking derivative on $s$ in (13) and letting $s \longrightarrow 0$, we arrive at (6) and (7). By a similar method, (5) can be obtained from (14).

Continuity condition (8) can be obtained from (13), and (10) can be obtained by comparing (13) with (14). (9) can be obtained by using (6) and (7), while (11) is derived by (5) and (6). Let $x=-c / \alpha$ in (5), and we have boundary condition (12).

Remark 1. Obviously, (6) and (7) are the same as (2.1) and (2.2) in Yuen et al. [3], respectively. 
2.2. Explicit Expressions of $v(x, b)$. In this section, the density function of claim sizes is supposed to be $f(x)=\mu e^{-\mu x}, x>0$. Applying the operator $(\mathrm{d} / \mathrm{d} x+\mu)$ to (5), then we have

$$
(\alpha x+c) \nu^{\prime \prime}(x, b)+(\alpha \mu x+c \mu+\alpha-\gamma-\sigma) \nu^{\prime}(x, b)-\sigma \mu \nu(x, b)=0,
$$

for $(-c / \alpha) \leq x<0$.

By the transforms $\nu(x, b)=k(z)$ and $z=-\mu(x+c / \alpha)$, (15) is reduced to a confluent hypergeometric equation:

$$
z k^{\prime \prime}(z)+\left(1-\frac{\sigma+\gamma}{\alpha}-z\right) k^{\prime}(z)+\frac{\sigma}{\alpha} k(z)=0, \quad z<0 .
$$

It follows from Abramowitz and Stegun [19] that $k(x)$ admits the following expression:

$$
\begin{aligned}
k(z)= & C_{1} e^{z} U\left(1-\frac{\gamma}{\alpha}, 1-\frac{\sigma+\gamma}{\alpha},-z\right)+C_{2} z^{(\sigma+\gamma / \alpha)} M \\
& \cdot\left(\frac{\gamma s}{\alpha}, 1+\frac{\sigma+\gamma}{\alpha}, z\right),
\end{aligned}
$$

where $C_{1}$ and $C_{2}$ are two constants, $M(a, b, z)$ is the first kind of confluent hypergeometric function, and $U(a, b, z)$ is the second kind of confluent hypergeometric function. Hence, the solution of (15) can be expressed as

$$
\begin{aligned}
\nu(x, b)= & C_{1} e^{-\mu(x+c / \alpha)} U\left(1-\frac{\gamma}{\alpha}, 1-\frac{\sigma+\gamma}{\alpha}, \mu\left(x+\frac{c}{\alpha}\right)\right) \\
& +C_{2}\left(-\mu\left(x+\frac{c}{\alpha}\right)\right)^{(\sigma+\gamma) / \alpha} M\left(\frac{\gamma}{\alpha}, 1+\frac{\sigma+\gamma}{\alpha},-\mu\left(x+\frac{c}{\alpha}\right)\right) .
\end{aligned}
$$

Due to boundary condition (13), one immediately deduces $C_{1}=0$.

Using a similar procedure to (15), we deduce

$$
c \nu^{\prime \prime}(x, b)-(\sigma+\gamma-c \mu) \nu^{\prime}(x, b)-\sigma \mu \nu(x, b)=0
$$

for $0<x<b$ and

$$
\begin{aligned}
& c \nu^{\prime \prime}(x, b)-(\gamma+\beta+\sigma-c \mu) \nu^{\prime}(x, b)-(\sigma+\beta) \mu \nu(x, b) \\
& \quad+\beta \mu[\nu(b, b)+x-b]+\beta=0,
\end{aligned}
$$

for $x \geq b$. Solving (19) and (20) by the knowledge of the ordinary differential equation, one deduces

$$
\begin{aligned}
& \nu(x, b)=C_{3} e^{\rho_{0} x}+C_{4} e^{R_{0} x}, \quad 0 \leq x<b, \\
& \nu(x, b)=D_{1} e^{\rho_{\beta} x}+D_{2} e^{R_{\beta} x}+D_{3} x+D_{4}, \quad x \geq b,
\end{aligned}
$$

where $\rho_{\beta}>0, R_{\beta}<0$ are roots of the equation

$$
r^{2}+\left(\mu-\frac{\sigma+\gamma+\beta}{c}\right) r-\frac{(\sigma+\beta) \mu}{c}=0 .
$$

Clearly, $v(x, b)$ is bounded. Hence, $D_{1}=0$. By (18), (21), and (22) and (8)-(11), we get the following equations:

$$
C_{3}+C_{4}=C_{2} \Delta_{1} \text {, }
$$

$$
C_{3} \rho_{0}+C_{4} R_{0}=C_{2} \Delta_{2}
$$

$$
D_{2} e^{R_{\beta} b}+D_{3} b+D_{4}=C_{3} e^{\rho_{0} b}+C_{4} e^{R_{0} b},
$$

$$
D_{2} R_{\beta} e^{R_{\beta} b}+D_{3}=C_{3} \rho_{0} e^{\rho_{0} b}+C_{4} R_{0} e^{R_{0} b}
$$

where

$$
\begin{aligned}
& \Delta_{1}=\left(\frac{-c \mu}{\alpha}\right)^{(\sigma+\gamma) / \alpha} M\left(\frac{\gamma}{\alpha}, 1+\frac{\sigma+\gamma}{\alpha},-\frac{c \mu}{\alpha}\right), \\
& \Delta_{2}=\frac{\sigma+\gamma}{c} \Delta_{1}-\frac{\gamma \mu}{\sigma+\alpha+\gamma}\left(\frac{-c \mu}{\alpha}\right)^{(\sigma+\gamma) / \alpha} M\left(1+\frac{\gamma}{\alpha}, 2+\frac{\sigma+\gamma}{\alpha},-\frac{c \mu}{\alpha}\right) .
\end{aligned}
$$

Substituting (22) into (7) and equating the coefficients of $x$ lead to

$$
D_{3}=\frac{\beta}{\beta+\sigma},
$$

and then equating the constant term yields

$$
\beta D_{2} e^{R_{\beta} b}-\sigma D_{4}=\left(\frac{\gamma}{\mu-c-\beta b}\right) D_{3}+\beta b .
$$

Solving (24)-(30), we get

$$
\begin{aligned}
& \nu(x, b)=\frac{\left(R_{0}-\rho_{0}\right) h(\beta)(-\mu(x+(c / \alpha)))^{\sigma+\gamma / \alpha} M((\gamma / \alpha), 1+(\sigma+\gamma / \alpha),-\mu(x+(c / \alpha)))}{H(\beta, b)}, \quad-\frac{c}{\alpha}<x<0, \\
& \nu(x, b)=\frac{\left(R_{0} \Delta_{1}-\Delta_{2}\right) h(\beta)}{H(\beta, b)} e^{\rho_{0} x}+\frac{\left(\Delta_{2}-\rho_{0} \Delta_{1}\right) h(\beta)}{H(\beta, b)} e^{R_{0} x}, \quad 0 \leq x \leq b, \\
& \nu(x, b)=D_{2} e^{R_{\beta} x}+D_{3} x+D_{4}, \quad x>b,
\end{aligned}
$$


where

$$
\begin{aligned}
D_{2} & =\frac{\left[\rho_{0}\left(R_{0} \Delta_{1}-\Delta_{2}\right) e^{\left(\rho_{0}-R_{\beta}\right) b}+R_{0}\left(\Delta_{2}-\rho_{0} \Delta_{1}\right) e^{\left(R_{0}-R_{\beta}\right) b}\right] h(\beta)}{R_{\beta} H(\beta, b)}-\frac{\beta}{R_{\beta}(\beta+\sigma)} e^{-R_{\beta} b}, \\
D_{3} & =\frac{\beta}{\beta+\sigma}, \\
D_{4} & =\frac{\left[\left(1-\left(\rho_{0} / R_{\beta}\right)\right)\left(R_{0} \Delta_{1}-\Delta_{2}\right) e^{\rho_{0} b}+\left(1-\left(R_{0} / R_{\beta}\right)\right)\left(\Delta_{2}-\rho_{0} \Delta_{1}\right) e^{R_{0} b}\right] h(\beta)}{H(\beta, b)}-\frac{\beta}{\beta+\sigma}\left(b-\frac{1}{R_{\beta}}\right), \\
H(\beta, b) & =\left[(\sigma+\beta) \rho_{0}-\sigma R_{\beta}\right]\left(\Delta_{2}-R_{0} \Delta_{1}\right) e^{\rho_{0} b}+\left[(\sigma+\beta) R_{0}-\sigma R_{\beta}\right]\left(\rho_{0} \Delta_{1}-\Delta_{2}\right) e^{R_{0} b}, \\
h(\beta) & =-\frac{\beta\left[\gamma R_{\beta}+\mu\left(\beta+\sigma-c R_{\beta}\right)\right]}{\mu(\beta+\sigma)} .
\end{aligned}
$$

Remark 2. For $\beta \longrightarrow \infty$, it is easy to check that $R_{\beta} \longrightarrow-\mu$. Letting $\beta \longrightarrow \infty$ in (31) and (32) and noticing $\rho_{0}+R_{0}=-\mu+(\sigma+\gamma) / c$, we have

$$
\begin{array}{ll}
V_{c}(x, b)=\frac{\left(\rho_{0}-R_{0}\right)(1+(\alpha x / c))^{(\sigma+\gamma) / \alpha} M(\gamma / \alpha, 1+(\sigma+\gamma / \alpha),-\mu(x+c / \alpha))}{\left(\rho_{0}+\mu\right) \Delta_{1}-(\gamma \mu / \gamma+\alpha+\sigma) M(1+(\gamma / \alpha), 2+(\sigma+\gamma / \alpha),-c \mu / \alpha)}, & -\frac{c}{\alpha}<x<0, \\
V_{c}(x, b)=\frac{\left(\Delta_{2}-R_{0} \Delta_{1}\right) e^{\rho_{0} x}+\left(\rho_{0} \Delta_{1}-R_{0}\right) e^{R_{0} x}}{\left(\rho_{0}+\mu\right) \Delta_{1}-(\gamma \mu / \gamma+\alpha+\sigma) M(1+(\gamma / \alpha), 2+(\sigma+\gamma / \alpha),-(c \mu / \alpha))}, & 0 \leq x \leq b,
\end{array}
$$

which are in accordance to (3.4) in Yuen et al. [3].

When the initial surplus $-c / \alpha<x \leq b$, we can investigate the optimal dividend barrier $b^{*}$ which can maximize $v(x, b)$ before absolute ruin. By (31) and (32), we identify that their numerators have nothing to do with the barrier $b$, and their denominators are $H(\beta, b)$. We also find that their numerators are bigger than 0 . So, the optimal barrier $b^{*}$ solves $H^{\prime}\left(\beta, b^{*}\right)=0$, i.e.,

$$
b^{*}=\frac{1}{\rho_{0}-R_{0}} \ln \frac{R_{0}\left[(\sigma+\beta) R_{0}-\sigma R_{\beta}\right]\left(\rho_{0} \Delta_{1}-\Delta_{2}\right)}{\rho_{0}\left[(\sigma+\beta) \rho_{0}-\sigma R_{\beta}\right]\left(R_{0} \Delta_{1}-\Delta_{2}\right)} .
$$

When $\beta \longrightarrow \infty, b^{*} \longrightarrow\left(1 / \rho_{0}-R_{0}\right) \ln \left(R_{0}^{2}\left(\rho_{0} \Delta_{1}-\Delta_{2}\right) /\right.$ $\left.\rho_{0}^{2}\left(R_{0} \Delta_{1}-\Delta_{2}\right)\right)$, which is the same as (3.5) in Yuen et al. [3].
Remark 3. When the claim sizes are general distributions, e.g., Erlang $(n)$ or mixture of exponential distribution, the explicit expression for $v(x, b)$ cannot be provided for $(-c / \alpha)<x<0$. Then, some numerical methods will be helpful, e.g., Yu et al. [20] and Zhang et al. [21].

\section{Results for $\Phi(x)$}

Using a similar method for Theorem 1, we can obtain the following results for the generalized Gerber-Shiu function $\Phi(x)$.

Theorem 2. The generalized Gerber-Shiu function $\Phi(x)$ admits the following expressions:

$$
\begin{gathered}
c \Phi^{\prime}(x)-(\gamma+\beta+\sigma) \Phi(x)+\beta \Phi(b)+\gamma s \int_{0}^{x+c / \alpha} \Phi(x-z) \varsigma(z) \mathrm{d} z+\gamma s \int_{x+c / x}^{\infty} \omega(x, z-x) \varsigma(z) \mathrm{d} z=0, \quad x \geq b, \\
c \Phi^{\prime}(x)-(\sigma+\gamma) \Phi(x)+\gamma s \int_{0}^{x+(c / \alpha)} \Phi(x-z) \varsigma(z) \mathrm{d} z+\gamma s \int_{x+(c / x)}^{\infty} \omega(x, z-x) \varsigma(z) \mathrm{d} z=0, \quad 0 \leq x<b,
\end{gathered}
$$




$$
(\alpha x+c) \Phi^{\prime}(x)-(\sigma+\gamma) \Phi(x)+\gamma s \int_{0}^{x+(c / \alpha)} \Phi(x-z) \varsigma(z) \mathrm{d} z+\gamma s \int_{x+(c / x)}^{\infty} \omega(x, z-x) \varsigma(z) \mathrm{d} z=0, \quad-\frac{c}{\alpha} \leq x<0
$$

with the following conditions:

$$
\begin{gathered}
\Phi(0+)=\Phi(0-), \\
\Phi(b+)=\Phi(b-), \\
\Phi^{\prime}(b+)=\Phi^{\prime}(b-), \\
\Phi^{\prime}(0+)=\Phi^{\prime}(0-),
\end{gathered}
$$

$$
\begin{array}{rr}
(\alpha x+c) \phi^{\prime \prime}(x)+(\alpha \mu x+c \mu+\alpha-\gamma-\sigma) \phi^{\prime}(x)+[\gamma \mu s-(\sigma+\gamma) \mu] \phi(x)=0, & -\frac{c}{\alpha}<x<0, \\
c \phi^{\prime \prime}(x)-(\sigma+\gamma-c \mu) \phi^{\prime}(x)-[(\sigma+\gamma) \mu-\gamma \mu s] \phi(x)=0, & 0 \leq x<b, \\
c \phi^{\prime \prime}(x)+(c \mu-\gamma-\beta-\sigma) \phi^{\prime}(x)-[(\gamma+\beta+\sigma) \mu-\gamma \mu s] \phi(x)+\beta \mu \phi(b)=0, & x \geq b .
\end{array}
$$

Then, solving the above system of equations leads to

$$
\begin{aligned}
& \phi(x)=c_{1} M_{1}(x, s)+c_{2} M_{2}(x, s), \quad-\frac{c}{\alpha} \leq x<0, \\
& \phi(x)=c_{3} e^{\rho_{0 s} x}+c_{4} e^{R_{0 s} x}, \quad 0 \leq x<b, \\
& \phi(x)=d_{1} e^{\rho_{\beta s} x}+d_{2} e^{R_{\beta s} x}+d_{4}, \quad x \geq b,
\end{aligned}
$$

where

$$
\begin{aligned}
M_{1}(x, s)= & e^{-\mu(x+(c / \alpha))} U\left(1-\frac{\gamma s}{\alpha}, 1-\frac{\sigma+\gamma}{\alpha}, \mu\left(x+\left(\frac{c}{\alpha}\right)\right)\right), \\
M_{2}(x, s)= & \left(-\mu\left(x+\left(\frac{c}{\alpha}\right)\right)\right)^{(\sigma+\gamma) / \alpha} M\left(\frac{\gamma s}{\alpha}, 1+\frac{\sigma+\gamma}{\alpha},-\mu\right. \\
& \left.\cdot\left(x+\left(\frac{c}{\alpha}\right)\right)\right),
\end{aligned}
$$

and $\rho_{\beta s}>0, R_{\beta s}<0$ are solutions of the equation

$$
c r^{2}+(\gamma+\beta+\sigma-c u) r-(\gamma+\beta+\sigma-\gamma s) \mu=0 .
$$

Since $\phi(x)$ is a bounded function, then $d_{1}=0$. By conditions (40)-(43), we have

$$
\begin{aligned}
\frac{\Gamma(\sigma+\gamma / \alpha)}{\Gamma(\gamma+\alpha+\sigma-\gamma s / \alpha)} c_{1} & =\frac{\gamma s}{\sigma+\gamma}, \\
c_{3}+c_{4} & =c_{1} M_{1}(0, s)+c_{2} M_{2}(0, s), \\
c_{3} \rho_{0 s}+c_{4} R_{0 s} & =c_{1} M_{1}^{\prime}(0, s)+c_{2} M_{2}^{\prime}(0, s), \\
c_{3} e^{\rho_{0 s} b}+c_{4} e^{R_{0 s} b} & =d_{2} e^{R_{\beta s} b}+d_{4}, \\
c_{3} \rho_{0 s} e^{\rho_{0 s} b}+c_{4} R_{0 s} e^{R_{0 s} b} & =d_{2} R_{\beta s} e^{R_{\beta s} b}
\end{aligned}
$$

Inserting (45) into (36) and comparing the coefficients of constants yield

$$
(\gamma s-\gamma-\sigma) d_{4}+\beta d_{2} e^{R_{\beta s} b}=0
$$

Solving (48)-(53), one obtains 


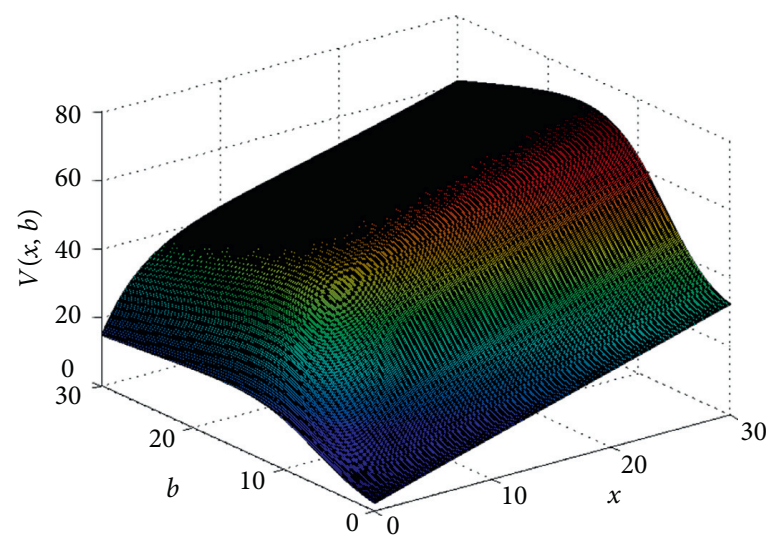

Figure 1: Influence of $x$ and $b$ on the expected discounted dividend $v(x, b)$.

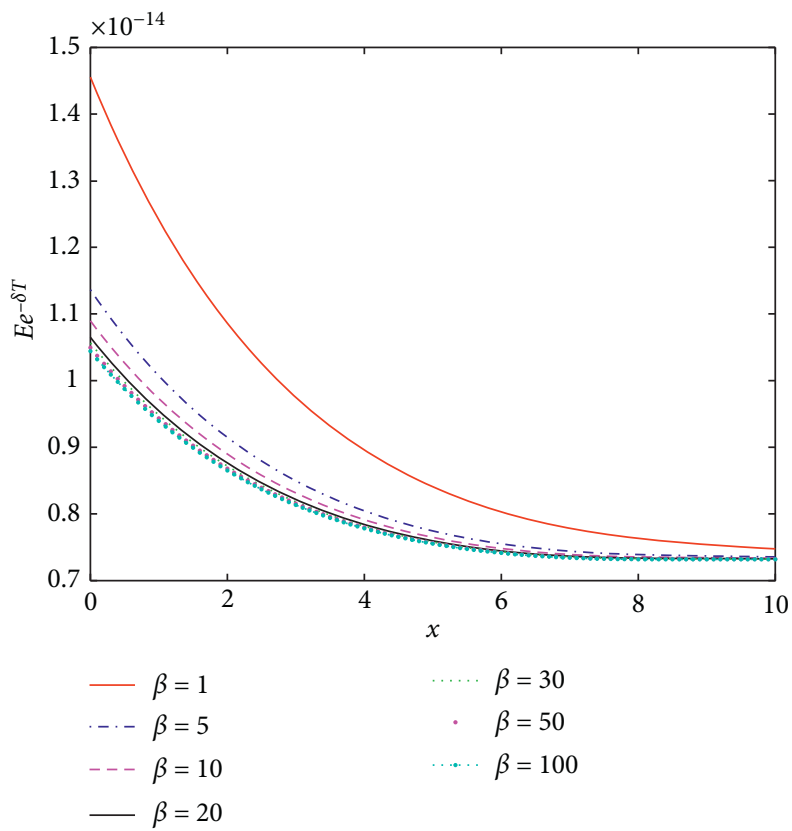

Figure 2: Influence of $\beta$ on $\mathbb{E}_{x}[\exp -\sigma T]$.

$$
\begin{aligned}
& c_{1}=\frac{\gamma s \Gamma(\gamma+\alpha+\sigma-\gamma s / \alpha)}{(\sigma+\gamma) \Gamma(\sigma+\gamma / \alpha)}, \\
& c_{2}=\frac{\gamma s \Gamma(\gamma+\alpha+\sigma-\gamma s / \alpha)\left[\left(\ell_{1}+\ell_{2}\right) M_{1}^{\prime}(0, s)+\left(\ell_{1} \rho_{0 s}+\ell_{2} R_{0 s}\right) M_{1}(0, s)\right]}{(\sigma+\gamma) \Gamma(\sigma+\gamma / \alpha)\left[\left(\ell_{1} \rho_{0 s}+\ell_{2} R_{0 s}\right) M_{2}(0, s)-\left(\ell_{1}+\ell_{2}\right) M_{2}^{\prime}(0, s)\right]}, \\
& c_{3}=\frac{\gamma s \Gamma(\gamma+\alpha+\sigma-\gamma s / \alpha) \ell_{1}\left[M_{1}^{\prime}(0, s) M_{2}(0, s)-M_{1}(0, s) M_{2}^{\prime}(0, s)\right]}{(\sigma+\gamma) \Gamma(\sigma+\gamma / \alpha)\left[\left(\ell_{1} \rho_{0 s}+\ell_{2} R_{0 s}\right) M_{2}(0, s)-\left(\ell_{1}+\ell_{2}\right) M_{2}^{\prime}(0, s)\right]}, \\
& c_{4}=\frac{\gamma s \Gamma(\gamma+\alpha+\sigma-\gamma s / \alpha) \ell_{2}\left[M_{1}^{\prime}(0, s) M_{2}(0, s)-M_{1}(0, s) M_{2}^{\prime}(0, s)\right]}{(\sigma+\gamma) \Gamma(\sigma+\gamma / \alpha)\left[\left(\ell_{1} \rho_{0 s}+\ell_{2} R_{0 s}\right) M_{2}(0, s)-\left(\ell_{1}+\ell_{2}\right) M_{2}^{\prime}(0, s)\right]}, \\
& d_{2}=\frac{\gamma s \Gamma(\gamma+\alpha+\sigma-\gamma s / \alpha)(\sigma+\gamma-\gamma s)\left[M_{1}^{\prime}(0, s) M_{2}(0, s)-M_{1}(0, s) M_{2}^{\prime}(0, s)\right]}{(\sigma+\gamma) \Gamma(\sigma+\gamma / \alpha)\left[\left(\ell_{1} \rho_{0 s}+\ell_{2} R_{0 s}\right) M_{2}(0, s)-\left(\ell_{1}+\ell_{2}\right) M_{2}^{\prime}(0, s)\right]} e^{-R_{\beta s} b}, \\
& d_{4}=\frac{\beta \gamma s \Gamma(\gamma+\alpha+\sigma-\gamma s / \alpha)\left[M_{1}^{\prime}(0, s) M_{2}(0, s)-M_{1}(0, s) M_{2}^{\prime}(0, s)\right]}{(\sigma+\gamma) \Gamma(\sigma+\gamma / \alpha)\left[\left(\ell_{1} \rho_{0 s}+\ell_{2} R_{0 s}\right) M_{2}(0, s)-\left(\ell_{1}+\ell_{2}\right) M_{2}^{\prime}(0, s)\right]},
\end{aligned}
$$




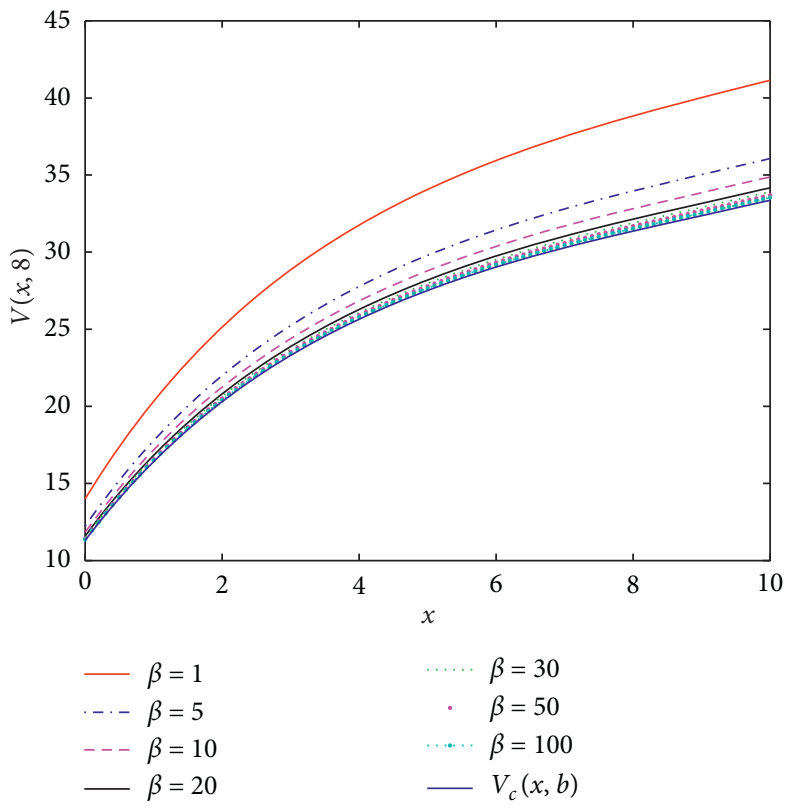

Figure 3: Influence of $\beta$ on $\nu(x, b)$.

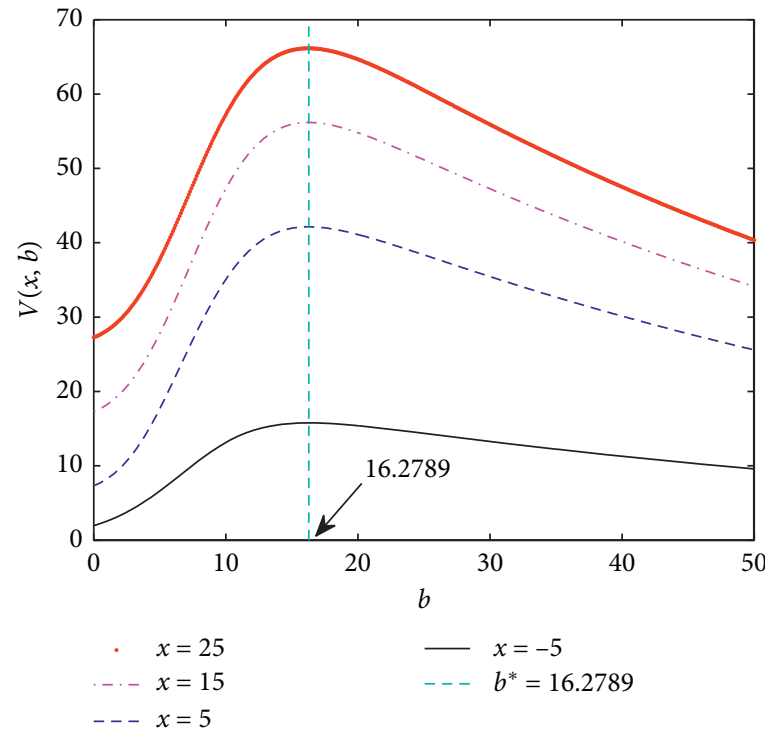

FIGURE 4: $v(x, b)$ as a function of $b$.

where

$$
\begin{aligned}
& \ell_{1}=\frac{\left(R_{0 s}-R_{\beta s}\right)(\sigma+\gamma-\gamma s)+\beta R_{0 s}}{R_{0 s}-\rho_{0 s}} e^{-\rho_{0 s} b}, \\
& \ell_{2}=\frac{\left(\rho_{0 s}-R_{\beta s}\right)(\sigma+\gamma-\gamma s)+\beta \rho_{0 s}}{\rho_{0 s}-R_{0 s}} e^{-R_{0 s} b} .
\end{aligned}
$$

\section{Numerical Examples}

In this last section, we give some numerical examples to illustrate the results. In Figures 1-4, we assume that the claim size follows exponential distribution with mean 2 , the premium rate $c=4$, the Poisson intensity $\gamma=0.8$, the discounted factor $\sigma=0.04$, and the debit interest $\alpha=0.06$. Figure 1 depicts the profiles of $\nu(x, b)$ for $(x, b) \in(0,30) \times$ $(0,30)$. We find that $v(x, b)$ is not a monotone function of $b$. When $b=8$, the curves of $\mathbb{E}_{x}\left[e^{-\sigma T} 1_{\{T<\infty\}}\right]$ and $\nu(x, b)$ are given in Figures 2 and 3 for several different $\beta$, respectively. It is shown that $\nu(x, b)$ is a decreasing function of $\beta$, while $\mathbb{E}_{x}\left[e^{-\sigma T} 1_{\{T<\infty\}}\right]$ is a increasing function of $\beta$. The bigger $\beta$ means the more frequent observation, which can lead to the earlier ruin. By Figure 3, we also find that $\nu(x, b)$ is more and more close to $V_{c}(x, b)$ as $\beta$ becomes more and more large. The profiles of $\nu(x, b)$ are given in Figure 4 for some different initial surplus $x$. By Figure 4, we know that the optimal barrier is $b^{*}=16.2789$ which has nothing to do with the initial surplus. 
TABLE 1: Impact of $\beta$ and $\alpha$ on $b^{*}$ when $c=4, \gamma=0.8, \mu=0.5$, and $\sigma=0.04$.

\begin{tabular}{lcccccccc}
\hline$\alpha$ & $\beta=1$ & $\beta=5$ & $\beta=10$ & $\beta=20$ & $\beta=30$ & $\beta=50$ & $\beta=100$ & $\beta \longrightarrow \infty$ \\
\hline 0.04 & 14.4939 & 15.9856 & 16.2774 & 16.4446 & 16.5045 & 16.5541 & 16.5925 & 16.6320 \\
0.06 & 14.4954 & 15.9870 & 16.2789 & 16.4460 & 16.5059 & 16.5556 & 16.5940 \\
0.07 & 14.4960 & 15.9877 & 16.2795 & 16.4467 & 16.5066 & 16.5563 & 16.5947 & 16.6335 \\
0.084 & 14.5125 & 16.0042 & 16.2960 & 16.4632 & 16.5231 & 16.5727 & 16.6111 & 16.6506 \\
\hline
\end{tabular}

The numerical results for $b^{*}$ are given in Table 1 for various observation intensity $\beta$ and debit interest $\alpha$. The optimal dividend barrier $b^{*}$ is an increasing function of $\beta$, which is also an increasing function of $\alpha$. More frequent observation will give rise to more dividend payments in the initial stage and earlier absolute ruin. In order to avoid the earlier absolute ruin, a bigger dividend barrier will be needed to decrease the dividend payments at the beginning.

\section{Data Availability}

No data were used to support this study.

\section{Conflicts of Interest}

The authors declare that they have no conflicts of interest.

\section{Acknowledgments}

This work was supported by the National Natural Science Foundation of China (nos. 11701319 and 11571198) and Fuyang Municipal Government-Fuyang Normal College Horizontal Cooperation Projects in 2017 (no. XDHXTD201709).

\section{References}

[1] H. U. Gerber and H. Yang, "Absolute ruin probabilities in a jump diffusion risk model with investment," North American Actuarial Journal, vol. 11, no. 3, pp. 159-169, 2007.

[2] J. Cai, "On the time value of absolute ruin with debit interest," Advances in Applied Probability, vol. 39, no. 2, pp. 343-359, 2007.

[3] K.-C. Yuen, M. Zhou, and J. Guo, "On a risk model with debit interest and dividend payments," Statistics \& Probability Letters, vol. 78, no. 15, pp. 2426-2432, 2008.

[4] C. Wang and C. Yin, "Dividend payments in the classical risk model under absolute ruin with debit interest," Applied Stochastic Models in Business and Industry, vol. 25, no. 3, pp. 247-262, 2009.

[5] J. Cai and H. Yang, "On the decomposition of the absolute ruin probability in a perturbed compound poisson surplus process with debit interest," Annals of Operations Research, vol. 212, no. 1, pp. 61-77, 2014.

[6] H. Albrecher, E. C. K. Cheung, and S. Thonhauser, "Randomized observation periods for the compound poisson risk model: dividends," ASTIN Bulletin, vol. 41, no. 2, pp. 645-672, 2011.

[7] B. Avanzi, E. C. K. Cheung, B. Wong, and J.-K. Woo, "On a periodic dividend barrier strategy in the dual model with continuous monitoring of solvency," Insurance: Mathematics and Economics, vol. 52, no. 1, pp. 98-113, 2013.
[8] Z. Zhang and E. C. K. Cheung, "The markov additive risk process under an erlangized dividend barrier strategy," Methodology and Computing in Applied Probability, vol. 18, no. 2, pp. 275-306, 2016.

[9] Z. Zhang and E. C. K. Cheung, "A note on a lévy insurance risk model under periodic dividend decisions," Journal of Industrial and Management Optimization, vol. 13, no. 2, pp. 36-46, 2018.

[10] B. Avanzi, V. Tu, and B. Wong, "On optimal periodic dividend strategies in the dual model with diffusion," Insurance: Mathematics and Economics, vol. 55, no. 1, pp. 210-224, 2014.

[11] H. Dong, C. Yin, and H. Dai, "Spectrally negative lévy risk model under erlangized barrier strategy," Journal of Computational and Applied Mathematics, vol. 351, pp. 101-116, 2019.

[12] X. Peng, W. Su, and Z. Zhang, "On a perturbed compound poisson risk model under a periodic threshold-type dividend strategy," Journal of Industrial \& Management Optimization, vol. 13, no. 5, pp. 1-20, 2017.

[13] H. U. Gerber and E. S. W. Shiu, "On the time value of ruin," North American Actuarial Journal, vol. 2, no. 1, pp. 48-72, 1998.

[14] S. Li and Y. Lu, "On the time and the number of claims when the surplus drops below a certain level," Scandinavian Actuarial Journal, vol. 2016, no. 5, pp. 420-445, 2016.

[15] W. Wang, P. Chen, and S. Li, "Generalized expected discounted penalty function at general drawdown for lévy risk processes," Insurance: Mathematics and Economics, vol. 91, pp. 12-25, 2020.

[16] A. D. Egidio dos Reis, "How many claims does it take to get ruined and recovered?" Insurance: Mathematics and Economics, vol. 31, no. 2, pp. 235-248, 2002.

[17] D. C. M. Dickson, "The joint distribution of the time to ruin and the number of claims until ruin in the classical risk model," Insurance: Mathematics and Economics, vol. 50, no. 3 , pp. 334-337, 2012.

[18] I. Czarna, Y. Li, Z. Palmowski, and C. Zhao, "The joint distribution of the parisian ruin time and the number of claims until parisian ruin in the classical risk model," Journal of Computational and Applied Mathematics, vol. 313, pp. 499-514, 2017.

[19] M. Abramowitz and I. A. Stegun, Handbook of Mathematical Function: With Formulas, Graphs, and Mathematical Tables, US Government Printing Office, Washington, DC, USA, 1972.

[20] W. Yu, Y. Yong, G. Guan, Y. Huang, W. Su, and C. Cui, "Valuing guaranteed minimum death benefits by cosine series expansion," Mathematics, vol. 7, no. 9, p. 835, 2019.

[21] Z. Zhang, Y. Yong, and W. Yu, "Valuing equity-linked death benefits in general exponential lévy models," Journal of Computational and Applied Mathematics, vol. 365, Article ID 112377, 2020. 